\title{
Multisite observations of the pre-Main-Sequence $\delta$ Scuti star IP Per
}

\author{
V. Ripepi ${ }^{1}$, S. Bernabei ${ }^{2,3}$, M. Marconi ${ }^{1}$, F. Palla ${ }^{4}$, \\ A. Arellano Ferro ${ }^{5}$, A. Bonanno ${ }^{6}$, P. Ferrara ${ }^{1}$, A. Frasca ${ }^{6}$, X.J. Jiang ${ }^{7}$, \\ S.L. Kim $^{8}$, S. Marinoni ${ }^{2}$, G. Mignemi ${ }^{6}$, T.D. Oswalt ${ }^{9}$, P. Reegen ${ }^{10}$, \\ J. Rimas ${ }^{11}$, E. Rodriguez ${ }^{12}$, A. Rolland ${ }^{12}$, A. Ruoppo ${ }^{13}$, \\ L. Terranegra ${ }^{1}$ and K. Zwintz ${ }^{10}$ \\ ${ }^{1}$ INAF-Osservatorio Astronomico di Capodimonte, Via Moiariello 16, 80131, Napoli, Italy \\ ${ }^{2}$ INAF-Osservatorio Astronomico di Bologna, Via Ranzani 1, 40127 Bologna, Italy \\ ${ }^{3}$ Departimento de Astrofísica, Universidad de La Laguna, Avda. Astrofisico F. Sánchez sn, \\ 30071 La Laguna, Spain \\ ${ }^{4}$ INAF-Osservatorio Astrofisico di Arcetri, Largo E. Fermi, 5, I-50125 Firenze, Italy \\ ${ }^{5}$ Instituto de Astronomía, UNAM, Apdo. Postal 70-264, México D.F., CP 04510, México \\ ${ }^{6}$ INAF-Osservatorio Astrofisico di Catania, Città Universitaria, 95125 Catania, Italy \\ ${ }^{7}$ National Astronomical Observatories, Chinese Academy of Sciences, Beijing, 100012, China \\ ${ }^{8}$ Korea Astronomy Observatory, Daejeon 305-348, Korea \\ ${ }^{9}$ Florida Inst. Technology, 150 W Univ. Blvd., Melbourne, FL 32901-6988, USA \\ ${ }^{10}$ Institute for Astronomy (IfA), University of Vienna, Türkenschanzstrasse 17, A-1180 Vienna \\ ${ }^{11}$ Institute of Theoretical Physics and Astronomy, Astronomical Observatory, Gostauto 12, \\ Vilnius LT-2600, Lithuania \\ ${ }^{12}$ Instituto de Astrofísica de Andalucía, CSIC, Apdo. 3004, 18080 Granada, Spain \\ ${ }^{13}$ Dipartimento di Scienze Fisiche, Università Federico II, Complesso Monte S. Angelo, 80126, \\ Napoli, Italy
}

\begin{abstract}
We present preliminary results of a photometric multisite campaign on the $\delta$ Scutitype Pre-Main-Sequence star IP Per. Nine telescopes have been involved in the observations, with a total of about 173 hour of observations over around 40 nights. Our current data permitted us to confirm the multiperiodic nature of this star and to determine at least 9 pulsational frequencies. A preliminary nonradial theoretical analysis seems to show that the star pulsates in a mixture of $l=0,1,2$ modes.
\end{abstract}

Keywords. Stars: Pre-Main-Sequence, stars: oscillations (including pulsations), stars: variables: $\delta$ Scuti, stars: interiors, stars: individual: (IP Per)

\section{Introduction}

Stellar pulsation is a very useful tool to investigate the internal structure and evolution of stars. This kind of analysis has been adopted for many MS and post-MS stars. In the last few years, new evidence showed that the intermediate mass Pre-Main-Sequence (PMS) Herbig Ae stars pulsate while crossing the Instability Strip for $\delta$ Scuti stars (see the review by Ripepi \& Marconi 2004). For more details on pulsation in PMS stars, see Bernabei et al. (2005), Marconi \& Palla (2005), and Zwintz et al. (2005). Now we will concentrate on the detailed photometric study of IP Per.

IP Per is a Herbig Ae star with: $V=10.34$, sp. type A7V, $\log L / L_{\odot} \sim 1.00 \pm 0.05$, and $T_{\text {eff }} \sim 8000 \pm 200 \mathrm{~K}$ (Miroshnichenko et al. 2001). With these values of the stellar 

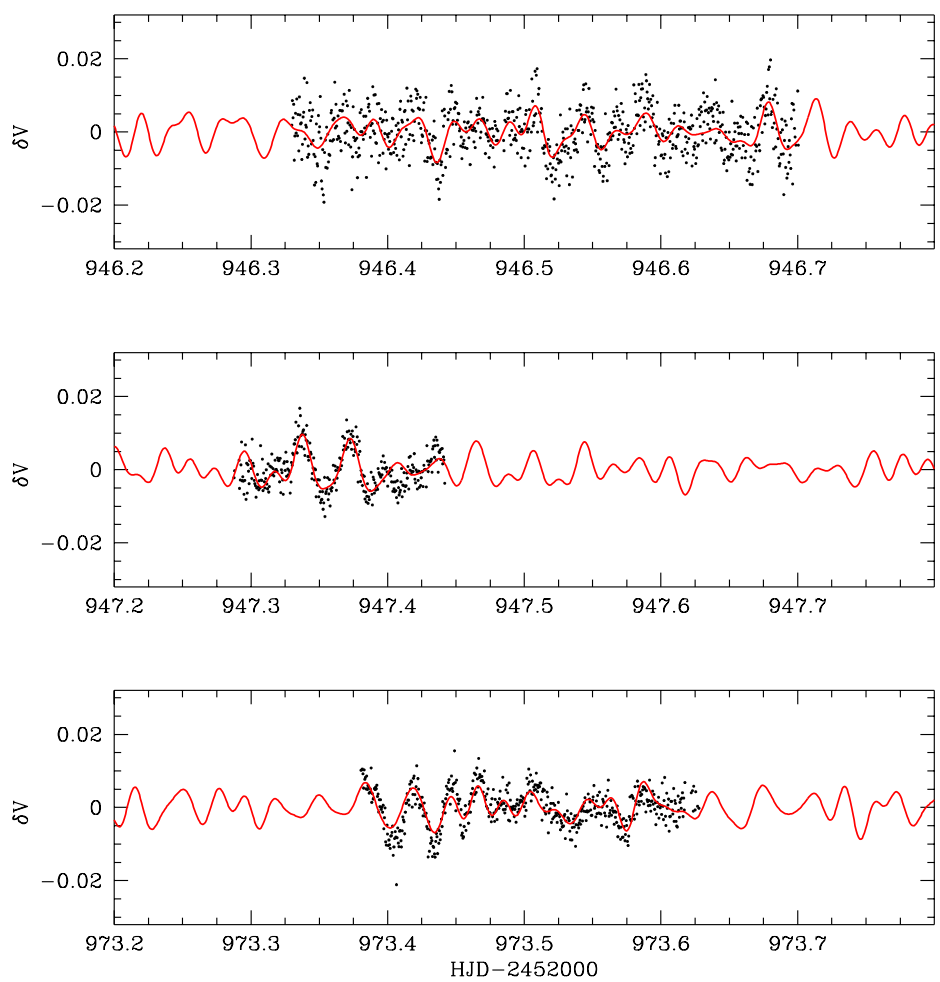

Figure 1. Data collected in Loiano with the TTCP during Nov./Dec. 2003 (dots). The solid line shows the fit to the data with 9 frequencies obtained from the frequency analysis.

parameters, IP Per falls very close to the blue edge of the theoretical instability strip for $\delta$ Scuti pulsation studied by Marconi \& Palla (1998). An exploratory observing run during 2002/2003 revealed that IP Per indeed pulsates as expected, showing a multiperiodic behaviour with 6 significant frequencies (Ripepi \& Marconi 2004). To overcome the well known one day alias problem, we decided to organize a multisite photometric campaign for fall-winter 2003. Preliminary results of this campaign are presented.

Table 1. List of telescopes and instruments involved in the multisite campaign.

\begin{tabular}{lll}
\hline Observatory & Telescope & Instrument \\
\hline Loiano (Italy) & $1.5 \mathrm{~m}$ & CCD - 2002/2003 data \\
Loiano (Italy) & $1.5 \mathrm{~m}$ & Three Channel Photometer \\
Beijing Astronomical Observatory (China) & $0.85 \mathrm{~m}$ & Three Channel Photometer \\
San Pedro Martir - SPM (Mexico) & $1.5 \mathrm{~m}$ & Danish (uvby) Photometer \\
Kitt Peak National Observatory (USA) & $0.9 \mathrm{~m}$ SARA & $512 \times 512$ CCD \\
Teide (Spain) & $1.0 \mathrm{~m}$ OGS & $1024 \times 1024$ CCD \\
Fairborn (USA) & $0.75 \mathrm{~m}$ APT & PMT \\
Sierra Nevada (Spain) & $0.9 \mathrm{~m}$ & $($ uvby) Photometer \\
Serra la Nave (Italy) & $0.9 \mathrm{~m}$ & PMT \\
SOAO (Korea) & $0.6 \mathrm{~m}$ & CCD \\
\hline & &
\end{tabular}




\section{Observations and data reduction.}

The campaign on IP Per can be divided in two parts, 1) single site observations during winter 2002/2003 (Loiano telescope) and 2) multisite campaign during winter 2003/2004, involving 9 different telescopes as listed in Table 1 . A total of about 173 hour of observations were obtained over about 40 nights. Each dataset has been reduced using the proper procedures for the three types of instruments available: CCD, single channel PMT, and three channel PMT. The dataset from each telescope was carefully inspected. Values due to bad weather (very frequent for PMT data) or system faults have been removed.

Due to differences among the various instruments (CCD, PMT) and filters used, and to prevent problems with consequent zero point variations between different datasets, we decided to detrend the data to a common average zero value. Of course, this procedure does not allow us to investigate low frequencies (roughly $\sim 3 \mathrm{~d}^{-1}$ ).

Thus we have obtained three different time series, 1) B-filter data from Loiano 2002/2003 observations, 2) B-filter data from the multisite campaign 2003/2004, and 3) V-filter data from the multisite campaign 2003/2004.

To show the quality of the data obtained in 2003 from the multisite campaign, we present in Figure 1 the observations collected in Loiano with the three channel photometer.
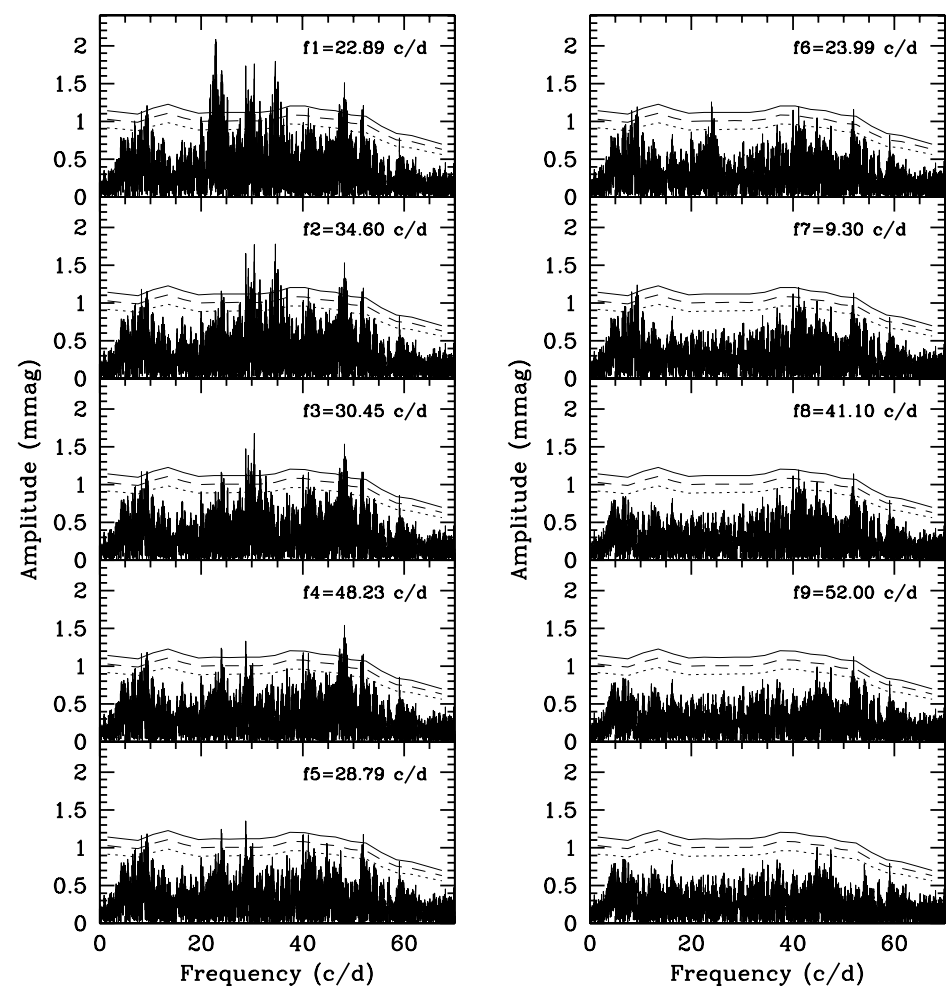

Figure 2. Fourier analysis of the V data of the 2003/2004 campaign. Each panel reports the periodogram after prewithening for the labeled frequency. The solid, dashed and dotted lines show the $99.9 \%, 99 \%$ and $90 \%$ confidence levels respectively. 




Figure 3. Position of the variable IP Per in the HR diagram according to the spectroscopic measurements of Miroshnichenko et al. (2001) (filled dot). The dashed region is the predicted Instability Strip for the first three radial modes by Marconi \& Palla (1998), whereas the black lines are the PMS evolutionary tracks computed with the FRANEC code (see Chieffi \& Straniero 1989) for the labeled masses. The open symbol is the selected PMS evolutionary model which was perturbed for nonradial pulsation.

\subsection{Frequency analysis}

The frequency analysis has been carried out using Period98 (Sperl 1998). Each data set described in the previous section has been analysed separately and the results crosschecked. On the basis of this analysis, we have found 9 significant frequencies which are shown in Figure 2 (campaign 2003/2004, V-filter). Here, each panel shows the periodogram after prewithening for the labeled frequency. The solid, dashed and dotted lines show the $99.9 \%, 99 \%$ and $90 \%$ confidence levels (see e.g., Breger et al. (1993) and Kuschnig et al. (1997)).

\section{Comparison with theory: preliminary results}

We tried an asteroseismological interpretation of the data using the Aarhus adiabatic nonradial pulsation code (http://astro.phys.au.dk/ jcd/adipack.n/). In particular, we run the FRANEC stellar evolution code (Chieffi \& Straniero 1989) to construct PMS evolutionary tracks in the range 1.6-2.0 $M_{\odot}$ and compared the resulting positions in the HR diagram with the position of IP Per based on the spectroscopic measurements of Miroshnichenko et al. (2001). As shown in Figure 3, this determination is intermediate between the evolutionary track for $1.7 M_{\odot}$ and $1.8 M_{\odot}$, but slightly in better agreement with the latter. We then applied the Aarhus adiabatic pulsation code to evolutionary models located along the $1.8 M_{\odot}$ PMS track and within the empirical uncertainty box of the spectroscopic determination. The output frequencies were compared with the observed periodicities leading to the best fit solution shown in Figure 3, which corresponds to a model likely pulsating in a mixture of $l=0,1,2$ modes. This preliminary analysis 
needs to be refined and a systematic detailed investigation of the dependence of theoretical results on the model properties is in progress. Finally, the position of IP Per in the HR diagram indicates a mass around $1.8 M_{\odot}$. Therefore this object could represent an ideal target to test the theoretical study by Suran et al. (2001) who investigated possible discriminating properties between preMS and postMS structures with $1.8 M_{\odot}$ on the basis of nonradial pulsational analysis.

\section{References}

Bernabei, S., Ripepi, V., Marconi, M., et al. 2005, These Proceedings, GP13

Breger, M., Stich, J., Garrido, R., Martin, B., Jiang, S.Y., Li, Z.P., Hube, D.P., Ostermann, W., Paparo, M., Scheck, M. 1993, A\&A 271, 482

Chieffi, A. \& Straniero, O. 1989, ApJS 71, 47

Kuschnig, R., Weiss, W.W., Gruber, R., Bely, P.Y., Jenkner, H. 1997, A\&̋A 328, 544

Marconi, M. \& Palla, F. 1998, ApJ 507, L141

Marconi, M. \& Palla, F. 2005, These Proceedings, 69

Miroshnichenko, A. S., Bjorkman, K. S., Chentsov, E. L., Klochkova, V. G., Gray, R. O., GarcíaLario, P.; Perea Calderón, J.V. 2001, A\&3A 377, 854

Ripepi, V. \& Marconi, M. 2004, in: F. Favata, S. Aigrain \& A. Wilson (eds.), Second Eddington Workshop: Stellar structure and habitable planet finding, 9 - 11 April 2003, Palermo, (Noordwijk: ESA Publications Division), p. 397

Sperl, M. 1998, CoAst 111, 1

Suran, M., Goupil, M., Baglin, A., Lebreton, Y., Catala, C. A\&SA 372, 233

Zwintz, K., Marconi, M., Weiss, W. W. 2005, These Proceedings, 353 